\title{
AUTHOR INDEX \\ Volume 60
}

Abdul-Hakim, Roslan, see Abdul-Razak, Nor Azam

4 (2015) 1550021

Abdullah, Abdul Jabbar, Doucouliagos, Hristos \& Manning,

Elizabeth, Is There a Kuznets' Process in Southeast Asia?

$2(2015) 1550017$

Abdul-Razak, Nor Azam, Karim, Mohd Zaini Abd \& Abdul-

Hakim, Roslan, Does Trade-Off Between Child Quantity and

Child Quality Exist in Malaysia?

$4(2015) 1550021$

Abeysinghe, Tilak, Lessons of Singapore's Development for Other

Developing Economies

3 (2015) 1550029

Afonso, Oscar \& Alves, Rui Henrique, Economic Growth Effects of an International Crisis

2 (2015) 1550012

Alves, Rui Henrique, see Afonso, Oscar

Anwar, Sofia, see Nasreen, Samia

2 (2015) 1550012

4 (2015) 1550061

Apergis, Nicholas \& Eleftheriou, Sofia, Renewable Energy

Consumption, Political and Institutional Factors: Evidence from

a Group of European, Asian and Latin American Countries

Apergis, Nicholas, see Omay, Tolga

1 (2015) 1550008

2 (2015) 1550018

Ariffin, Tengku Faekah Tengku, see Awang-Hashim, Rosna

4 (2015) 1550027

Asher, Mukul G., Bali, Azad Singh \& Kwan, Chang Yee, Public

Financial Management in Singapore: Key Characteristics and

Prospects

3 (2015) 1550032

Awang-Hashim, Rosna, Lim, Hock Eam, Yatim, Bidin, Ariffin,

Tengku Faekah Tengku, Zubairi, Ainol Madziah, Yon, Haniza \&

Osman, Omar, Estimating a Prediction Model for the Early

Identification of Low Employability Graduates in Malaysia

4 (2015) 1550027

Aysan, Ahmet F., Disli, Mustafa, Ozturk, Huseyin \& Turhan,

Ibrahim M., Are Islamic Banks Subject to Depositor Discipline?

$1(2015) 1550007$

Aysan, Ahmet Faruk \& Bilgin, Mehmet Huseyin, Introduction and

Editorial Overview

$1(2015) 1502001$

Aysan, Ahmet Faruk, Fendoğlu, Salih \& Kılınç, Mustafa,

Macroprudential Policies as Buffer Against Volatile

Cross-Border Capital Flows

1 (2015) 1550001

Bakar, Normizan, see Hasan-Basri, Bakti

5 (2015) 1550048 
Bali, Azad Singh, see Asher, Mukul G.

3 (2015) 1550032

Batten, Jonathan A., Morgan, Peter \& Szilagyi, Peter G., Time

Varying Asian Stock Market Integration

1 (2015) 1550006

Bhaskaran, Manu, see Tan, Kim Song

3 (2015) 1550030

Bilgin, Mehmet Huseyin, Gozgor, Giray \& Karabulut, Gokhan,

The Impact of World Energy Price Volatility on Aggregate

Economic Activity in Developing Asian Economies

1 (2015) 1550009

Bilgin, Mehmet Huseyin, see Aysan, Ahmet Faruk

1 (2015) 1502001

Bilgin, Mehmet Huseyin, see Danis, Hakan

1 (2015) 1550005

Chang, Youngho, Energy and Environmental Policy

3 (2015) 1550039

Chen, Ku-Hsieh, see Lee, Joe-Ming

5 (2015) 1550117

Chia, Ngee-Choon, Adding a Basic Pillar to the Central Provident

Fund System: An Actuarial Analysis

3 (2015) 1550037

Chia, Ricky Chee Jiun, Lim, Shiok Ye, Ong, Pui Khuan \& Teh,

Siew Fong, Pre and Post Chinese New Year Holiday Effects:

Evidence from Hong Kong Stock Market

4 (2015) 1550023

Chia, Siow Yue, Globalization and Regionalization: Singapore's

Trade and FDI

3 (2015) 1550034

Ch'ng, Kean Siang \& Khoo, Suet Leng, Market Mechanisms to Allocate Heritage Conservation Fund: An Experimental Study

Cho, Chin-Ho, see Lee, Joe-Ming

Choi, Yong-Seok \& Min, Insik, Cash Holdings with the

Precautionary Motive Among Korean Chaebol and Non-Chaebol

Firms: A Quantile Regression Approach

5 (2015) 1550047

Chor, Foon Tang \& Eu Chye Tan, The Relative Contributions of

Domestic and Foreign Direct Investments and Exports to

Malaysia's Economic Growth

5 (2015) 1550024

5 (2015) 1550117

Danis, Hakan, Demir, Ender \& Bilgin, Mehmet Huseyin,

Conditional Jump Dynamics in Stock Returns: Evidence from

MIST Stock Exchanges

$1(2015) 1550005$

Demir, Ender, see Danis, Hakan

Disli, Mustafa, see Aysan, Ahmet F.

1 (2015) 1550005

1 (2015) 1550007

Doucouliagos, Hristos, see Abdullah, Abdul Jabbar

2 (2015) 1550017

Ekinci, Cagdas, see Ozdemir, Zeynel Abidin

4 (2015) 1550087

Eleftheriou, Sofia, see Apergis, Nicholas

Ergun, Selim Jürgen, Centrist's Curse? An Electoral Competition

1 (2015) 1550008

Model with Credibility Constraints

5 (2015) 1550055

Eu, Chye Tan, see Chor, Foon Tang

2 (2015) 1550011

Fendoğlu, Salih, see Aysan, Ahmet Faruk

1 (2015) 1550001

Francisco, Jamil Paolo S., Willingness to Pay for Air Quality

Improvements from Using Electric Jeepneys in Metro Manila

4 (2015) 1550073

Gee, Christopher, see Yap, Mui Teng

3 (2015) 1550035

Gokmenoglu, Korhan, see Ozdemir, Zeynel Abidin

4 (2015) 1550087 
Goy, Siew Ching \& Johnes, Geraint, Differences in Decline:

Quantile Regression of Male-Female Earnings Differential in Malaysia

4 (2015) 1550054

Gozgor, Giray, see Bilgin, Mehmet Huseyin

1 (2015) 1550009

Gupta, Abhijit Sen, Extent of Exchange Rate Coordination in Asia

2 (2015) 1550013

Hasan-Basri, Bakti, Karim, Mohd Zaini Abd \& Bakar, Normizan, Willingness to Pay for Recreational Attributes of Public Parks: A Choice Experiment Approach

5 (2015) 1550048

Hasan, Iftekhar, Raymar, Steven \& Song, Liang, Effects of

Corporate and Country Governance on $R \& D$ Investment:

Evidence from Emerging Markets

1 (2015) 1550003

Hassan, M Kabir, see Lee, Doowon

4 (2015) 1550092

Huang, Hsin-Yi, see Huang, Li-Hsuan

4 (2015) 1550022

Huang, Li-Hsuan \& Huang, Hsin-Yi, Rigid Low College Premiums and the Expansion of Higher Education in Taiwan

4 (2015) 1550022

Inoue, Takeshi, Central Bank Intervention and Exchange Rate

Behavior: Empirical Evidence for India

2 (2015) 1550016

John, Joice, Has Inflation Persistence in India Changed Over

Time?

Johnes, Geraint, see Goy, Siew Ching

Kam, Andrew Jia-Yi, Determinants of International Production

Fragmentation: Malaysia's Experience in the Information,

Communications and Telecommunications (ICT) Industry

Karabulut, Gokhan, see Bilgin, Mehmet Huseyin

5 (2015) 1550042

1 (2015) 1550009

Karim, Mohd Zaini Abd, see Abdul-Razak, Nor Azam

Karim, Mohd Zaini Abd, see Hasan-Basri, Bakti

4 (2015) 1550021

5 (2015) 1550048

Khoo, Suet Leng, see Ch'ng, Kean Siang

5 (2015) 1550024

1 (2015) 1550001

Kim, Youngkyu, Oh, Inha \& Lee, Jeong-Dong, Economic Impact Assessment of Public-Private Matching Fund Programs Using Firm-Level Data

Kong, Dongmin, see $\mathrm{Xu}$, Jin

4 (2015) 1550060

5 (2015) 1550062

3 (2015) 1550032

5 (2015) 1550041

Lar, Ni, see Taguchi, Hiroyuki

Lau, Chi Keung Marco, Yang, Fu Steve, Zhang, Zhe \& Leung,

Vincent K. K., Determinants of Innovative Activities: Evidence from Europe and Central Asia Region

Lean, Hooi Hooi, see Ting, Irene Wei Kiong

Lee, Doowon, Hassan, M Kabir \& Rahman, M Arifur, Firm Specific Variation in Returns and Fundamentals in Korea Stock Market

4 (2015) 1550092

Lee, Jeong-Dong, see Kim, Youngkyu

1 (2015) 1550004

2 (2015) 1550019

4 (2015) 1550060 
Lee, Joe-Ming, Chen, Ku-Hsieh \& Cho, Chin-Ho, The Relationship Between $\mathrm{CO}_{2}$ Emissions and Financial Development: Evidence from OECD Countries

5 (2015) 1550117

Lee, Soo Ann, Governance and Economic Change in Singapore 3 (2015) 1550028

Leung, Vincent K. K., see Lau, Chi Keung Marco

1 (2015) 1550004

Lim, Chong Yah, Economic Growth and Exchange Rate: A New Road Ahead for Indonesia

2 (2015) 1550010

Lim, Hock Eam, see Awang-Hashim, Rosna

Lim, Linda Y. C., Beyond Gender: The Impact of Age, Ethnicity,

4 (2015) 1550027 Nationality and Economic Growth on Women in the Singapore Economy

2 (2015) 1550020

Lim, Linda Y. C., Fifty Years of Development in the Singapore Economy: An Introductory Review

Lim, Linda Y. C., see Pang, Eng Fong

Lim, Shiok Ye, see Chia, Ricky Chee Jiun

Limpanonda, Suphannada, Provincial Disparities, Convergence and Effects on Poverty in Thailand

Manning, Elizabeth, see Abdullah, Abdul Jabbar

Manzoor, Rabia, see Samad, Ghulam

Min, Insik, see Choi, Yong-Seok

Miyake, Yusuke, see Watanabe, Minoru

Morgan, Peter, see Batten, Jonathan A.

Morita, Keisuke, Advance Tax Payments and Tax Evasion: A Note

Mourmouras, Alexandros \& Rangazas, Peter, International

Lending and the Samaritan's Dilemma

3 (2015) 1502002

3 (2015) 1550033

4 (2015) 1550023

2 (2015) 1550015

2 (2015) 1550017

2 (2015) 1550014

5 (2015) 1550047

5 (2015) 1550043

1 (2015) 1550006

5 (2015) 1450050

1 (2015) 1550002

Nasreen, Samia, Anwar, Sofia \& Waqar, Masood Qadir, Institutions, Investment and Economic Growth: A Cross-Country and Panel Data Study

$4(2015) 1550061$

$\mathrm{Ng}$, Irene Y. H., Being Poor in a Rich "Nanny State":

Developments in Singapore Social Welfare

$\mathrm{Ng}$, Yew-Kwang, Some Conceptual and Methodological Issues on Happiness: Lessons from Evolutionary Biology

Oh, Inha, see Kim, Youngkyu

Omay, Tolga, Apergis, Nicholas \& Özçelebi, Hülya, Energy

Consumption and Growth: New Evidence from a Non-linear

Panel and a Sample of Developing Countries

2 (2015) 1550018

Ong, Pui Khuan, see Chia, Ricky Chee Jiun

4 (2015) 1550023

Osman, Omar, see Awang-Hashim, Rosna

4 (2015) 1550027

Özçelebi, Hülya, see Omay, Tolga

2 (2015) 1550018

Ozdemir, Zeynel Abidin, Ekinci, Cagdas \& Gokmenoglu, Korhan, International Evidence on Real Interest Rate Persistence

4 (2015) 1550087

Ozturk, Huseyin, see Aysan, Ahmet F.

1 (2015) 1550007 
Paweenawat, Sasiwimon Warunsiri, see Vechbanyongratana, Jessica

5 (2015) 1550082

Phang, Sock-Yong, Singapore's Housing Policies: Responding to the Challenges of Economic Transitions

3 (2015) 1550036

Pang, Eng Fong \& Lim, Linda Y. C., Labor, Productivity and

Singapore's Development Model

Rahman, M Arifur, see Lee, Doowon

3 (2015) 1550033

4 (2015) 1550092

Rangazas, Peter, see Mourmouras, Alexandros

1 (2015) 1550002

Raymar, Steven, see Hasan, Iftekhar

1 (2015) 1550003

Samad, Ghulam \& Manzoor, Rabia, Green Growth: Important

Determinants

2 (2015) 1550014

Song, Liang, see Hasan, Iftekhar

1 (2015) 1550003

Szilagyi, Peter G., see Batten, Jonathan A.

1 (2015) 1550006

Taguchi, Hiroyuki \& Lar, Ni, Fragmentation and Trade of

Machinery Parts and Components in Mekong Region

5 (2015) 1550041

Tan, Kim Song \& Bhaskaran, Manu, The Role of the State in

Singapore: Pragmatism in Pursuit of Growth

Teh, Siew Fong, see Chia, Ricky Chee Jiun

Ting, Irene Wei Kiong \& Lean, Hooi Hooi, Does Government

Ownership Matter? Comparative Study Between GLCs and

NGLCs in Malaysia

2 (2015) 1550019

1 (2015) 1550007

Vechbanyongratana, Jessica \& Paweenawat, Sasiwimon Warunsiri, Transfer Payments and Upper Secondary School Outcomes: The Case of Low-Income Female Students in Thailand

Waqar, Masood Qadir, see Nasreen, Samia

Watanabe, Minoru, Miyake, Yusuke \& Yasuoka, Masaya, Public Investment Financed by Consumption Tax in an Aging Society

3 (2015) 1550030

4 (2015) 1550023

Wilson, Peter, Monetary Policy and Financial Sector Development

$\mathrm{Xu}$, Jin \& Kong, Dongmin, Understanding the Household

Consumption Behavior in Urban China

Yang, Fu Steve, see Lau, Chi Keung Marco

Yap, Mui Teng \& Gee, Christopher, Singapore's Demographic

Transition, the Labor Force and Government Policies: The Last

Fifty Years

3 (2015) 1550035

Yasuoka, Masaya, see Watanabe, Minoru

5 (2015) 1550043

Yatim, Bidin, see Awang-Hashim, Rosna

4 (2015) 1550027

Yon, Haniza, see Awang-Hashim, Rosna

4 (2015) 1550027

Zhang, Zhe, see Lau, Chi Keung Marco

1 (2015) 1550004

Zubairi, Ainol Madziah, see Awang-Hashim, Rosna

4 (2015) 1550027 